\title{
Evaluation of the early radiological and functional results of percutaneous fixation of unstable pelvic ring injuries
}

M.I.Shaaban, S.M.Zahid, A.S.Rizk and S.A.Shoulah

\author{
Orthopedic, Dept., Faculty of Medicine, Benha Univ., Benha, Egypt
}

E-mail: Mohamed Shaaban @gmail.com

\begin{abstract}
Recent years have seen improvements in all phases of pelvic fracture management. The surgical treatment of pelvic ring injuries is technically demanding The aim of treatment is to achieve anatomical or near anatomical restoration of the articular surface, accurate reduction of the pelvic ring in mechanically unstable injuries, reduce pain, allows early mobilization of the patient and optimizes long term functional results This study aimed to evaluate the early radiological and functional results of percutaneous fixation of unstable pelvic ring injuries. From October 2019 till December 2020 twenty patients (11male and 9 female) with unstable pelvic ring injuries were included in the study. The mean age 42 years (range 18-66 years). The most common mode of injury was road traffic accident (RTA) followed by fall from height. The most common injury pattern according to Young and Burgess lateral compression injury, anteroposterior compression injury and vertically unstable. Four patients had medical comorbidities diabetes mellitus and hypertension at presentation. All patients treated by DCO protocol. 3 patients with posterior pelvic ring injuries treated by percutaneous iliosacral screw. 3 patients with anterior pelvic ring injuries treated by external fixator. 2 patient with anterior pelvic ring injuries treated by INFIX .8 patients with anterior and posterior pelvic ring injuries treated by external fixator and percutaneous iliosacral screw.1 patients with anterior and posterior pelvic ring injuries treated by INFIX and percutaneous iliosacral screw.2patients with anterior pelvic ring and posterior pelvic ring injuries treated by INFIX and spinopelvic fixation.1 patients with anterior pelvic ring and posterior pelvic ring injuries treated by external fixator and spinopelvic fixation. The mean injury-to-surgery interval was 6.1 days (range 0-12 days). The mean intraoperative blood loss $160 \mathrm{ml}$ range $100 \mathrm{ml}-240 \mathrm{ml}$. Percutaneous fixation of pelvic ring injuries offers safe, short operative time technique, less complication, with better results, short hospital stay, early healing and should be the standard way for pelvic ring fractures fixation. High quality pre-operative and intra-operative imaging, image-guidance, are essential for an equally high quality of surgical results.
\end{abstract}

Keyword: percutaneous, fixation, pelvic, Injury.

\section{Introduction}

The pelvis is a key component of the axial skeleton that links the lower extremities with the rest of the body through the lumbosacral spine. Its ring like structure allows it to surround and protect important inner organs such as the urinary bladder, the lower intestines, and the reproductive organs as well as major nerves and blood vessels. In patients with healthy bone high energy injuries are required to disrupt the integrity of the pelvic ring [1].

Because of the high energy injury mechanisms required to sustain these injuries in the younger population patients with pelvic fractures have a high likelihood of associated injury to the abdominopelvic structures including neurologic and vascular injuries which can result in permanent neurologic deficits as well as severe life threatening hemorrhage compared with other injuries death after pelvic ring fractures is high particularly because these injuries often occur in poly traumatized patients [2].

High energy pelvic fractures often disrupt the integrity of the anterior and/or posterior osteoligamentous structures thereby resulting in vertical or rotational instability of the pelvic ring. early restoration of the structural integrity and stability of the pelvic ring allow for early restoration of mobility, function, diminish bleeding, smooth the progress of rehabilitation, and prevent long-term complications related to mechanical instability [3].
The surgical treatment of pelvic ring injuries is technically demanding The aim of treatment is to achieve anatomical or near anatomical restoration of the articular surface, accurate reduction of the pelvic ring in mechanically unstable injuries, reduce pain, allows early mobilization of the patient and optimizes long term functional results [4].

Surgical stabilization of pelvic ring fractures can be achieved by open or percutaneous methods. Open methods of stabilization include lag screws, tension band plates and bars. They all provide a comparable biomechanical stability. The main advantage of open treatment is that it permits direct visualization and reduction of the fracture, permitting earlier mobilization of the patient and a shorter period of bed rest but it requires extensive surgical exposure. It is done with the patient in the prone position, often through severely traumatized soft tissues. Complications of the open surgical exposure frequently are worse than those of the pelvic injury itself. Infection rates of up to $27 \%$ and neurovascular complications can be expected. The pelvic hematoma is disrupted and additional blood loss occurs unequivocally so definite stabilization must be delayed until the soft tissue heal and the hematoma is mature [5].

Unstable pelvic ring injuries are also associated with high morbidity and mortality. It is therefore reasonable to explore options for fixation which allow adequate reduction and stabilization of the articular surface with minimal invasive surgical procedure. 
Percutaneous pelvic fixation has been receiving more attention in an attempt to avoid extensile surgical approaches .[6].

Unstable pelvic ring injuries require stabilization of anterior and posterior pelvic ring. Posterior pelvic ring stability can be restored by closed reduction and percutaneous iliosacral screws fixation and anterior pelvic ring injuries can be treated by either external fixator or anterior subcutaneous cross-over internal pelvic fixator ("Infix") which combine advantages and avoid disadvantages of external fixation frames and ORIF devices. Anterior stabilization restores the integrity of the anterior ring and prevents displacement around a posteriorly fixed pelvis. In unstable pelvic fractures with hemodynamic instability resulting from intrapelvic hemorrhage, emergent pelvic tamponade and stabilization can be accomplished with a temporary anterior external fixator as a "damage control" procedure which may later be converted to a definitive form of internal fixation [7].

Definitive anterior external fixation is preferred in the presence of urological injuries, presence of a suprapubic catheter, displaced but vertically stable lateral compression fractures, multiple comminuted pubic rami fractures, refractory coagulopathy, open pelvic fractures, Open book fracture and Bucket-handle fracture [8].

This study aimed to evaluate the early radiological and functional results of percutaneous fixation of unstable pelvic ring injuries.

\section{Patients and methods}

This study was a prospective clinical notes and radiological studies was performed on patients with unstable pelvic ring injuries treated by minimally invasive fixation in the form of percutaneous iliosacral screws in patients with posterior ring injuries and by external fixator or a novel anterior subcutaneous internal fixation device, "INFIX" in patient with anterior ring injuries treated at Benha university hospital, Menofia university hospital and Nasser Institute from October 2019 till December 2020 .. Twenty patients, 11 males and 9 females.

\section{- Inclusion criteria:}

- Age : adult older than 18 years old

- Sex: both sex

- Recent unstable pelvic ring injuries

\section{- Exclusion criteria:}

- Pathological fracture

- Associated acetabular fractures

Preoperative Management and Evaluation:

- Patient's history:

- Personal history: Patient name, age, sex, occupation, marital status, smoking, heavy activities.
- History of trauma: type of injury, high energy impact was the mechanism of injury. Stability of the injured pelvic ring was evaluated based on radiological appearances, physical findings and mechanism of injury.

\section{- Clinical examination:}

- General examination:

Assessment of patient general condition, consciousness, vital signs, and other systems review.

\section{- Local examination:}

Clinical assessment involved inspection of the undressed pelvis for hematomas, open wounds, variations of the external contour, and deformities of the pelvis and/or lower extremities (shortening, external rotation deformities). As well as an assessment of the gross neurovascular status. Per rectal (PR) and per vaginal $(\mathrm{PV})$ in non-virgin female examination were done to exclude rectal or vaginal injuries. Reduction of unstable pelvic fractures was held by a bed sheet tied around the pelvis.

\section{- $\quad$ Radiological Evaluation :}

Primary radiological evaluation consisted of at least an anterior posterior pelvic X-ray As long as the patient was stable, an additional views were taken as pelvic inlet and pelvic outlet views. CT was done (to better defines posterior injury, amount of displacement versus impaction, rotation of fragments and amount of comminution Retrograde urethrography was done in case of suspecting urethral injuries .

\section{- laboratory :}

Complete blood picture, blood glucose level, and renal function test, liver Function tests, prothrombin time, blood grouping and viral hepatitis markers were done for all patients.

\section{- Classification of pelvic fracture}

Young and Burgess Classification was applied to all cases.

\subsection{Methods of treatment:}

\section{Percutaneous iliosacral screws.}

\section{Indications:}

$>$ Disruptions of the SI joint in Tile type $\mathrm{C}$ pelvic injuries which can be reduced anatomically in closed technique.

$>$ Sacral fractures without concomitant nerve damage in Tile type $\mathrm{C}$ pelvic injuries which can be reduced anatomically in closed technique.

$>$ Fracture dislocations of the SI joint with insignificant small fragment which can be reduced anatomically in closed technique (177).

$>$ Severe soft tissue injury, bowel injury or a combination of both these injuries is a good indication because this technique avoids large exposure and the risk of infection.

Advantages:

$>$ Prone or supine position. Supine is an advantage as it allows simultaneous treatment of associated injuries of anterior part of pelvis. 
$>$ minimal exposure required, excellent stability of fixation, less risk of wound problems, especially infection, the operative and anaesthesia times are decreased, the blood loss is minimized.

$>$ Allows fixation in acute setting without exacerbation of haemorrhage .

$>$ Allows fixation in the presence of Morel Lavellee lesions.

\section{Surgical Technique:}

Percutaneous iliosacral screws may be placed with the patient in either the supine or prone position on a radiolucent table. Supine positioning is preferred by most surgeons. Soft support was placed underneath the lumbosacral spine to elevate the patient from the table. All patients receive bowel preparation the day before surgery to enable fluoroscopic visualization of the radiographic landmark

Fluoroscopic views: The $\mathrm{C}$-arm is used to visualize the lateral aspect of the sacrum. The AP view is now obtained by rotating the $\mathrm{C}$-arm. To obtain the inlet view, the $\mathrm{C}$ - arm is then tilted so that the anterior cortex of S1 overlaps that of S2. If such visualization is not done, the concavity of the sacrum will not be appreciated and the screw may exit anteriorly. However, the posterior cortex of $\mathrm{S} 1$ is best seen if the anterior cortex of $\mathrm{S} 1$ is over the coccyx. This projection is needed to ensure that the screw does not exit posteriorly. The outlet view is obtained by rotating the $\mathrm{C}$ arm so that the pubic tubercles lie just inferior to the $\mathrm{S} 1$ foramen and the symphysis overlies the midline of the sacrum. After complete radiographic visualization has been obtained, fracture reduction can be undertaken.

Closed reduction: is usually possible within 2 to 5 days of the injury. The first displacement to be corrected is the axial malposition, which is accomplished by longitudinal skeletal traction through a traction pin inserted in the distal end of the femur if the fracture is posteriorly displaced, the traction is directed upward to correct it. Rotational displacement is corrected by placing one or two Schanz screws into the involved iliac crest and using them to manipulate the hemipelvis into place (fig 38). The external fixator can be used to reduce this component as well. Posterior translational displacement is reduced by closing this gap with a ballspiked pusher. Depending on the desired vector of reduction force, the femoral distractor can be used. Anatomical reduction and fixation of the anterior ring may also be helpful in reduction.

Once the reduction is achieved, provisional fixation is possible with a $\mathrm{K}$-wire inserted into the ala or use of the pelvic resuscitation C- clamp.

The superficial skin location for screw insertion is $2 \mathrm{~cm}$ posterior to the intersection of a line from the femoral shaft and a line dropped from the anterior superior iliac spine. The guide wire or drill bit is placed through a stab wound down to the posterolateral aspect of the ilium. The AP view shows that this device is aimed into the $\mathrm{Sl}$ body and perpendicular to the sacroiliac joint. At this point, the C-arm is used to visualize the lateral part of the sacrum.
The position of the drill bit or guide wire is confirmed to be in the middle of the Sl body so that it is seen end-on. It is important to make sure that the screw is placed so that it is below the cortical projection of the sacral ala, which is seen only on the lateral view The Carm is then rotated to the anteroposterior plane. The insertion of the guide wire or drill bit is continued only after confirming a satisfactory direction on the inlet and outlet views. If the position is correct, the drill bit or guide wire is advanced toward the body of Sl. It is useful to halt insertion of the pin when the tip reaches the superior aspect of the lateral border of the first sacral foramen on the outlet view. A true lateral view of the sacrum is obtained to determine the relationship of the pin tip to the sacral alar slope and its anteroposterior position relative to the first sacral vertebral body; the pin tip should be located beneath (caudal to) the iliac cortical density and centered within the vertebral body

The progress of the drilling is watched on the three pelvic views. The drill bit or guide wire perforates three cortical barriers (outer part of the ilium, inner iliac side of the sacroiliac joint and sacral side of the sacroiliac joint). If a fourth cortical barrier is encountered insertion is stopped and the drill bit or guide wire is realigned because it is potentially about to leave the safe channel and injure a major structure. Any misdirected drill bits or guide wires must be completely removed and restarted to create a new tract. Once the position of the drill bit or guide wire is confirmed the screw is inserted.

\section{Anterior internal fixator (INFIX) General description:}

Modern subcutaneous internal fixators for anterior pelvic ring injuries combine advantages and avoid disadvantages of external fixation frames and ORIF devices. Anterior subcutaneous cross-over internal pelvic fixator ("Infix") fixators

Anterior subcutaneous cross-over internal pelvic

Consist of two large diameter (7-8 mm), long (75$110 \mathrm{~mm})$ pedicle screws inserted into bilateral supraacetabular bone and interconnected by a $5-6 \mathrm{~mm}$ curved, titanium or stainless steel rod.

The original description of the fixator was by Kuttner et al. who published an explanation of the surgical technique and mid-term clinical results in the German literature in 2009.

\section{Indications}

1-To restore the integrity and stability of anterior pelvic ring, in an unstable posterior pelvic ring fracture associated with additional anterior ring disruption (pubic rami fractures and symphysis disruption), which required stabilization:

$>$ vertically unstable injuries (AO/OTA type 61-C),

$>$ lateral compression injuries (AO/OTA type 61-B2),

$>$ Open book injuries (AO/OTA type 61-B3.1).

2- Unstable injuries of the anterior pelvic ring

$>$ Morbidly obese patients

$>$ Patients with severe soft tissue injuries 
$>$ Patients who require prolonged stay in the ICU to reduce risk of infection and facilitate nursing care

$>$ Patients with concomitant injuries that may require prone positioning for surgical procedures (e.g. spine fractures)

$>$ Patients with coagulopathy. Both techniques result in minimal intraoperative bleeding.

3- Isolated injuries of the anterior pelvic ring associated with incapacitating pain, particularly in elderly individuals, to provide immediate stability and facilitate rehabilitation

\section{Advantages:}

$>$ 1-They are meant to require minimal soft tissue dissection.

$>$ 2- Diminish associated blood loss and postoperative pain.

$>$ 3-Provide rigid fixation.

$>$ 4-stay remote from important visceral and neurovascular structures.

$>$ 5-remain subcutaneous to mitigate surgical site infection.

$>$ 6-facilitate nursing care.

$>$ 7- Avoid interference with the patient's rehabilitation and activities of daily living.

\section{Indications}

\section{External fixation:}

The use of external fixation is limited to:

$>$ Resuscitation.

$>$ Provisional stabilization for vertically and rotationally unstable injuries to help mobilize the patient for other tests and make him or her more comfortable in the early post-operative period.

$>$ Definitive stabilization for:

$\checkmark$ Rotationally unstable pelvis but vertically stable injuries (intact posterior tension band)

$\checkmark \quad$ For type $\mathrm{C}$ if the posterior internal fixation is not possible, in this case it should be supplemented by double symphyseal plating, or traction.

$\checkmark \quad$ For open pelvic fractures

$>$ (4)Open-book fracture

$\checkmark$ Bucket-handle fracture.

The advantages of external fixation are that it is minimally invasive, preserves the biology at the fracture site, can be removed easily, and is generally technically less demanding, requiring less operating room time and blood loss than ORIF.

\section{Technique:}

The normal orientation of the iliac wing is $45^{\circ}$ oblique to the sagittal and coronal body axes. Additionally, the iliac crest has a lateral overhang and the iliac fossa present a concave internal surface

Traditional guidelines have called for the placement of two to three iliac crest pins per side, but from the view point of stability use of pin clusters is questionable. One reliable pin in each innominate bone is sufficient for primary external fixation in the acute setting. A standard rectangular or triangular frame is all that is needed in most situations
Once a preliminary reduction is achieved, small incisions for the pins can be made. it is advantageous to use transverse incisions angled across the iliac crest at $90^{\circ}$ and directed toward the umbilicus. If a later iliac crest incision is planned, standard stab wounds parallel to the crest are preferred to minimize compromise of the incision by these wounds.

A small diameter smooth $\mathrm{K}$-wire can be placed along the medial aspect of the iliac crest to act as a directional finder for the drill and the pin.

When placing the pins, it is important to start the pin just medial to the midline of the iliac crest. After the starting point is identified, a drill bit is directed in the appropriate orientation as determined by the guide. A drill hole that just perforates the iliac crest is made. The size of the drill hole is determined by the system in use. Normally, for 5-mm pins, a $3.2-$ to $3.5-\mathrm{mm}$ drill bit is required.

Once the pin has been seated in the predrilled hole, it is gently turned by hand and allowed to seek its way between the inner and outer cortical tables of the innominate bone at an orientation approximately perpendicular to the crest.

The complete threads of the pin should be buried within the iliac bone. Once the pins have been placed into both hemipelves, they can be used cautiously as a handle to assist in reduction of the hemipelvis.

If the patient's condition permits, pin placement should be checked before the patient leaves the operating room. The outlet view reveals whether the pin is out of the crest. The obturator oblique view is tangential to the crest and shows whether the pins are between the two tables. A combination of both views provides excellent visualization of the iliac crest to guide insertion.

If multiple iliac crest pins are utilized, the most anterior pin is positione $\mathrm{cm}$ dorsal to the anterior superior iliac spine to avoid the lateral femoral cutaneous nerve

Supra-acetabular pin placement offers some advantages with regard to soft tissue clearance and conversion to definitive open surgery where appropriate; however, it does require fluoroscopic control in most cases.

A pilot smooth K-wire is inserted approximately 5 $\mathrm{cm}$ distal and 2 to $3 \mathrm{~cm}$ lateral to the ASIS. This ideally should localize a starting point approximately $2 \mathrm{~cm}$ proximal to the hip joint and slightly lateral to the AIIS.

This position is verified using combined obturator outlet and iliac inlet views, and the $\mathrm{K}$-wire is seated with a mallet.

Once the starting point is established a small longitudinal incision is made and a drill/pin sleeve is passed over the guide wire. A drill bit is then passed to create an aperture directed posteriorly toward the greater sciatic notch. The exact trajectory is determined by the anticipated needs for hip flexion and abdominal clearance. The pin is then inserted by hand through the retained sleeve. 
After pin placement and verification the frame can be constructed. A simple frame with adequate thigh and abdominal clearance is the goal and can be achieved with a variety of different constructs.

Once the frame has been applied, final fluoroscopically controlled adjustments in the reduction are carried out and the frame is tightened.

\subsection{Postoperative management:}

\section{A-Medications:}

1. Antibiotics: broad spectrum injection antibiotics were given with induction of anesthesia, and continued for 7 days then oral for 7 days or more according presence of hematoma, wound dressing, associated injuries.

2. Analgesics: Strong analgesics started immediately postoperative and non morphia analgesics when needed.

3. omeprazole $20 \mathrm{mg}$ : $150 \mathrm{mg}$ twice daily was given to all patients preoperatively and continued postoperative to guard against post-operative gastric troubles. Foley catheters were removed once the patient regained control of his bladder activity. Prophylactic antibiotics during this period were taken.

4. Anti-edematous, anti-inflammatory and neuroprotective drugs.

\section{B- Postoperative Care}

Low-molecular-weight heparin was administered subcutaneously to all patients post injury to the day of discharge for prophylaxis against deep vein thrombosis. Postoperatively, active hip and knee joint motion was encouraged. Non weight bearing with under arm crutches was allowed as soon as possible according to the general condition. Partial weight bearing was allowed at 4-6 weeks and full weight bearing usually began at 12-14 weeks postoperatively. All patients received antithrombotic prophylaxis for 6 weeks. Ease of nursing, patient mobility and comfort were observed during hospital stay. Patients could sit up from second day onwards depending on their general condition, associated injuries, functional status and pain tolerance.

\subsection{Follow-up}

Patients were followed up at monthly intervals for the first three months bimonthly up to one year. At each follow-up patients' general condition a detailed neurological examination including the femoral nerve and LFCN ease of mobility during daily activities quality of reduction and implant position on radiographs (anteroposterior, inlet and outlet views of pelvis) and complications. The quality of fracture reduction was evaluated according to Matta's radiologic criteria.

Patients were allowed toe-touch walking at 2 4 weeks partial weight bearing at 6-8 weeks and full weight bearing at $8-10$ weeks post-surgery. Removal of External fixator and INFIX device was planned once the fracture showed radiological signs of healing. Functional outcomes and return to work status were assessed using Majeed score (excellent: score of 95 or more, good: 85-94, fair: 70-84, poor: <70).

\section{Results}

This study was conducted in orthopedic surgery department, faculty of medicine, Benha university hospitals on twenty patients (11male and 9 female) with unstable pelvic ring injuries were included in the study. The mean age $42 \pm 24$ years (range $18-66$ years). The most common mode of injury was road traffic accident (RTA) followed by fall from height. The most common injury pattern according to Young and Burgess lateral compression injury, anteroposterior compression injury and vertically unstable. Four patients had medical comorbidities diabetes mellitus and hypertension at presentation Table (1)

\subsection{Operative considerations}

In the present study, All patients treated by DCO protocol .3 patients with posterior pelvic ring injuries treated by percutaneous iliosacral screw. 3 patients with anterior pelvic ring injuries treated by external fixator. 1 patient with anterior pelvic ring injuries treated by INFIX .8 patients with anterior and posterior pelvic ring injuries treated by external fixator and percutaneous iliosacral screw.1 patients with anterior and posterior pelvic ring injuries treated by INFIX and percutaneous iliosacral screw.3patients with anterior pelvic ring and posterior pelvic ring injuries treated by INFIX and spinopelvic fixation.1 patients with anterior pelvic ring and posterior pelvic ring injuries treated by external fixator and spinopelvic fixation. The mean injury-tosurgery interval was $6.1+/$ _ 3.1 days (range $0-12$ days). The mean intraoperative blood loss $160 \mathrm{ml}$ range 100_240 ml.

\subsection{Radiological outcome}

Status of fracture healing and adequacy of reduction Fracture healing was seen at a mean postoperative period of 3.5 months (range 3-6 months). The results were classified according to Matta's criteria. Into the following groups. Excellent $(0-4 \mathrm{~mm}$ displacement), good (4-10 mm displacement), fair (10 $20 \mathrm{~mm}$ displacement) and poor (>20 mm displacement). Reduction was excellent in fourteen patients, good in five patients and fair in one patient at final follow-up, according to Matta's criteria.

\subsection{Functional outcomes and return to work}

Functional outcomes were excellent in twelve patients, good in seven patients and fair in one patient as assessed by Majeed score .eighteen patients had returned to their pre-injury jobs, while two had to change their jobs involving lighter duties.

Table (1) Demographic characteristics in study population.

\begin{tabular}{lll}
\hline & \multicolumn{3}{l}{ General characteristics } \\
\hline Age (years) & Mean \pm SD & $42 \pm 24$ \\
Gender & Male $\quad$ n (\%) & $11(55 \%)$ \\
& Female $\quad$ n (\%) & $9 \quad(45 \%)$ \\
\hline
\end{tabular}


Table (2) This table shows radiological results of all included patients according to maximal residual posterior displacement.

\begin{tabular}{lcc}
\hline Outcome & $\mathbf{N}$ & $\%$ \\
\hline Excellent & $\mathbf{1 4}$ & $\mathbf{7 0 \%}$ \\
Good & 5 & $\mathbf{2 5 \%}$ \\
Fair & 1 & $5 \%$ \\
Total & $\mathbf{2 0}$ & $\mathbf{1 0 0 \%}$ \\
\hline
\end{tabular}

Table (3) This table shows clinical and functional results of all included patients according to Majeed score at final follow up.

\begin{tabular}{lll}
\hline Majeed score & N & \% \\
\hline excellent & $\mathbf{1 2}$ & $\mathbf{6 0 \%}$ \\
Good & $\mathbf{7}$ & $\mathbf{3 5 \%}$ \\
Fair & $\mathbf{2 0}$ & $\mathbf{5 \%}$ \\
Total & $\mathbf{2 0}$ & $\mathbf{1 0 0 \%}$ \\
\hline
\end{tabular}

\section{Discussion}

The advantages of external fixation are that it is minimally invasive, preserves the biology at the fracture site, can be removed easily, and is generally technically less demanding, requiring less operating room time and blood loss than ORIF But, anterior external fixation is associated with several complications including pin site infections $(2-50 \%)$, osteomyelitis $(0-7 \%)$, aseptic loosening (0-19\%), loss of reduction (0-33\%), frame bulkiness hindering patient mobilization, difficulties in nursing care, and hindrance to wound access or surgical access to abdomen [9].

So to overcome the complications of anterior external fixation, to improve patient comfort and facilitate early mobilization, several innovative techniques of minimally invasive anterior pelvic ring stabilization have been described in recent years .The "INFIX" is one such technique which utilizes the already established principles of external fixation. Biomechanical studies have shown that the INFIX has superior stability with respect to axial stiffness and stiffness at pubic symphysis, in the management of vertically and rotationally unstable pelvic ring injuries [10].

In the largest series published by Vaidya et al., INFIX was used to treat lateral compression fractures, anteroposterior compression fractures, and vertical shear or combined fracture patterns [11].

Hoskins et al. used INFIX for lateral compression and anteroposterior compression fractures [12].Scheyerer et al. used minimally invasive INFIX as an alternative to external fixation/anterior plating, in pelvic fractures with concomitant abdominal injuries necessitating multiple abdominal surgeries [13].

Recently, INFIX has also been used in cases of open pelvic injuries. They are meant to require minimal soft tissue dissection, diminish associated blood loss and postoperative pain, provide rigid fixation, stay remote from important visceral and neurovascular structures, remain subcutaneous to mitigate surgical site infection, facilitate nursing care and avoid interference with the patient's rehabilitation and activities of daily living. We addressed posterior ring instability first similar to the case series reported by Vaidya et al. and Hoskins et al [12].

On the other hand, Gardner et al. performed anterior fixation prior to posterior fixation [14].

Posterior pelvic ring stability can be restored by closed reduction and percutaneous iliosacral screws fixation in these criteria of patient within 5 days of injury, Preoperatively, the pelvis has been placed in traction to correct displacement, Severe soft tissue injury, bowel injury, or a combination of both these injuries is a good indication because this technique avoids large exposure and the risk of infection.

This technique is contraindicated if Failure to reduce the fracture by closed means, Failure to visualize with a $\mathrm{C}$-arm and the presence of sacral dimorphism

From October 2019 till December 2020 twenty patients (11male and 9 female) with unstable pelvic ring injuries were included in the study. In this study males were affected more than females. This can be explained by over activity of males and more exposure to transportation .this nearly agree with the finding of Dujardin et al [15].

In the present study, young adults were more affected. This can be explained by young adults are at high risk of high energy trauma as they are highly active, ambitious and hasty during driving. In addition they have strong body built that can withstand the major pelvic trauma until their primary admission. That is similar to the findings of Lindahl and Hirvensalo et al. [16].

In this study road traffic accidents was the main causative trauma (65\% of all causative trauma) of patients. This can be explained by the fact that road traffic accidents were a high energy trauma as compared to other causative agents. Similar findings had been found in other studies as that of Miranda et al. [17].

In this study the most common associated injuries were extremity fractures followed by abdominal injuries retroperitoneal hemorrhage $(n=2)$, Anal tear $(n=1)$, splenic rupture $(n=1)$, spine injuries $(n=2)$. Two 
patients had neurological deficit at presentation due to associated spinal injuries.

In this study hospital stay and healing time were statistically evident shorter Ayoub M.A. reported that iliosacral screwing during the first week had strong impact on the hospital stay duration and it has lowered significantly the needed total amount of transfused blood before and after its application [18].

In this study status of fracture healing and adequacy of reduction Fracture healing was seen at a mean post-operative period of 3.5 months (range 3-6 months). Reduction was excellent in fourteen patients, good in five patients and fair in one patient at final follow-up, according to Matta's criteria.

In this study Functional outcomes were excellent in twelve patients, good in seven patients and fair in one patient as assessed by Majeed score .eighteen patients had returned to their pre-injury jobs, while two had to change their jobs involving lighter duties

In this study postoperative residual displacement more than $1 \mathrm{~cm}$ (unsatisfactory radiological results) was associated with unsatisfactory clinical results. This agrees with the series of McLaren et al [19].Whereas postoperative residual displacement less than $1 \mathrm{~cm}$ (satisfactory radiological results) was associated with satisfactory clinical results. This agrees with the study of Lindalh and Hirvensalo [16].who described that an excellent radiographic result showed a clear association with excellent or good functional outcome.

In a retrospective clinical study, Rommens et al reported that satisfactory radiological and clinical results were not only related to stability and symmetry of the pelvic ring, but also dependent on the severity and amount of damage to the soft tissues around the pelvis [20].

Miranda et al. showed that there were no differences in functional outcome across fracture types or treatment methods, and questioned whether reduction of pelvic ring injuries could alter patient long-term functional outcome [17].

In this study three patient had unilateral anterolateral thigh paresthesia due to LFCN compression which recovered following implant removal. Two patients had early superficial surgical site infection within a week after surgery (probably due to the associated urinary tract infection) which settled with administration of culture specific antibiotics. One patient had screw loosening and loss of fixation which need revision.one patients had transient L5 neuropraxia last about 6 month. The incidence of LFCN injury was less $(1 / 20)$ in comparison with studies published thus far, possibly due to our meticulous approach during dissection, taking care to protect the LFCN from injury due to our awareness of this complication from previous studies. We ensured that the screw head remained above deep fascia to prevent compression of femoral nerve and inguinal ligament. We used $7 \mathrm{~mm}$-diameter polyaxial pedicle screws in comparison to that used by Vaidya et al. (7- to 8.5-mm), Muller et al. (6- to 7-mm), Gardner et al. (7- or 7.5-mm) and Hoskins et al. $(10 \mathrm{~mm})$ [11].
We recommended hardware removal to our patients after radiological evidence of healing of anterior and posterior ring fractures. External fixator was removed at mean postoperative periods ranging from 2 to 4 months. In comparison with previous studies where INFIX was removed at mean postoperative periods ranging from 3 to 9.4 months, in our series, the INFIX was removed after a mean postoperative period of 7.3+/ _ 1.5 months (5.5-11 months). The combination of INFIX and percutaneous iliosacral screws or external fixator and percutaneous iliosacral screws is a minimally invasive, biomechanically stable and effective surgical technique for definitive management of vertically and/or rotationally unstable pelvic ring injuries. The technique is easy to learn and apply, achieves good fracture reduction with minimum complications and offers good functional and radiological outcomes with high patient satisfaction and return to preinjury level function.

\section{Conclusion}

Percutaneous fixation of pelvic ring injuries offers safe, short operative time technique, less complication, with better results, short hospital stay, early healing and should be the standard way for pelvic ring fractures fixation. High quality pre-operative and intra-operative imaging, image-guidance, are essential for an equally high quality of surgical results.

\section{References}

[1] Z. Balogh ,K. King, P. Mackay . The epidemiology of pelvic ring fractures: a population-based study.vol.6,pp.72-73,2007.

[2] B.Gabbe, R.deSteiger, M. Esser,Predictors of mortality following severe pelvic ring fracture: results of a population-based study.vol.42,pp.985-991,2011.

[3] O.Pieske ,C. Landersdorfer , C.Trumm , CTguided sacroiliac percutaneous screw placement in unstable posterior pelvic ring injuries: accuracy of screw position, injury reduction and complications in 71 patients with 136 screws.vol.46,pp.333-339,2015.

[4] M. Failinger ,P. McGainty, Current concepts review_ unstable fractures of the pelvic ring. J Bone Joint Surg.vol.8,pp.74-81,1992.

[5] J. Kellam , R.McMurtry , D.Paley, The unstable pelvic fracture: operative treatment. Orthop Clin North Am.vol.18,pp.25-41,1987.

[6] D.Barei , C.Bellabarba , W. Mills ,J. Routt ME . Percutaneous management of unstable pelvic ring disruptions.vol.3,pp.33-44,2001.

[7] B.Riemer, S.Butterfield, D. Diamond,Acute mortality associated with injuries to the pelvic ring: the role of early patient mobilization and external fixation.vol.35,pp.66-79,1993.

[8] C. Bellabarba , W.Ricci , B. Bolhofner , Distraction external fixation in lateral compression pelvic fractures.vol.14,pp.475482,2000 . 
[9] W.Mason, S.Khan ,C. James , T. Chesser ,A. Ward , Complications of temporary and definitive external fixation of pelvic ring injuries. Injury.vol.36,pp.599-604,2005.

[10]E. Mcdonald ,A. Theologis , P. Horst ,U. Kandemir ,M. Pekmezci ,When do anterior external or internal fixators provide additional stability in an unstable (Tile C) pelvic fracture?. Eur J Trauma .vol.41,pp.665671,2015

[11]R.Vaidya ， E.Kubiak ， P. Bergin ,Complications of anterior subcutaneous internal fixation for unstable pelvis fracturesClin Orthop Relat Res.vol.70,pp.21242131,2012.

[12] W.Hoskins ,A. Bucknill , J.Wong, A prospective case series for a minimally invasive internal fixation device for anterior pelvic ring fractures.vol.15,pp.111-135,2016.

[13] M. Scheyerer ,S. Zimmermann , G.Osterhoff , S.Tiziani ,H. Simmen , G.Wanner , Anterior subcutaneous internal fixation for treatment of unstable pelvic fractures. BMC Res Notes .vol.4,pp.17-33,2014.

[14]M. Gardner , S.Mehta ,A. Mirza ,W. Ricci , Anterior pelvic reduction and fixation using a subcutaneous internal fixator. J Orthop Trauma.vol.26,pp.314-321,2012.

[15]F.Dujardin , M.Duparc , F.Biga ,N. Thomine, Long-term functional prognosis of posterior injuries in high-energy pelvic disruption. J Orthop Trauma.vol.12,pp.145-150,1998.

[16] J. Lindahl , E. Hirvensalo, Outcome of operatively treated type-C pelvic ring injuries ActaOrthap.vol.76,pp.667- 678,2005.

[17]M.Miranda ， B.Riemer ,C. Butterfield , C.Burke, Pelvic ring injuries: A long-term functional outcome study. Chin Orthop.vol.6,pp.29-52,1996.

[18] M.Mostafa, Type C pelvic ring injuries in polytrauma patients: can percutaneous iliosacral screws reduce morbidity and costs? Eur J Orthop Surg.vol.22,pp.137-144,2012.

[19] A.McLaren , C.Rorabeck ,J. Halpenny .Longterm pain and disability in relation to residual deformity after displaced pelvic ring fractures..vol.3,pp.42-94,1990.

[20]P.Rommens ,M. Hessmann, Staged reconstruction of pelvic ring disruption: differences in morbidity, mortality, radiologic and functional outcomes between B1, B2/B3, and C-type lesions.vol.16,pp.2989,2002 . 